\title{
The Frequency of Phospholipid Antibodies in an Unselected Stroke Population
}

\author{
L.M. Metz, S. Edworthy, R. Mydlarski and M.J. Fritzler
}

\begin{abstract}
Background: Antibodies to cardiolipin and other phospholipids have been associated with recurrent thrombotic events, including stroke. Methods: Over a 16 month period we assessed an unselected cohort of 151 ischemic stroke patients for the presence of antiphospholipid antibodies. Patients with known systemic lupus erythematosis, systemic sclerosis, or Sjögrens Syndrome were excluded. Sera from patients admitted to hospital with a diagnosis of ischemic stroke $(n=151)$ and from controls $(n=111)$ assessed during the same period were tested for antiphospholipid antibodies (APLA) using 3 assays; anticardiolipin antibodies (ACA) by ELISA, prolonged activated partial thromboplastin time (APTT), and VDRL. Results: The average age of ischemic stroke cases was 68 years (range 29 to 91) and of controls 63 years (range 29 to 86). The prevalence of APLA detected by at least one of the three methods was $12 \%$ for IS cases and $10 \%$ for controls. After correcting for known risk factors such as age, gender, diabetes mellitus, heart disease, hypertension, and smoking, the odds ratio for risk of stroke fell to 0.8 (C.I. 0.4 to 1.2). Conclusions: Our findings suggest that APLA may not be an independent risk factor for ischemic stroke in unselected persons who do not have known systemic lupus erythematosis or systemic sclerosis but further evaluation of the role of lupus anticoagulant is indicated.
\end{abstract}

RÉSUMÉ: La prévalence d'anticorps antiphospholipides dans une population de patients non sélectionnés ayant subi un accident vasculaire cérébral. Introduction: Les anticorps dirigés contre la cardiolipine et d'autres phospholipides ont été associés à des événements thrombotiques récurrents, dont la thrombose cérébrale. Méthodes: Nous avons évalué la présence d'anticorps antiphospholipides (AAPL) dans une cohorte de 151 patients ayant présenté un accident vasulaire cérébral (AVC) sur une période de 14 mois. Les patients qui avaient un lupus érythémateux aigu disséminé, une sclérodermie systémique ou un syndrome de Sjögrens ont été exclus. Pendant cette période, nous avons mesuré les AAPL dans le sérum de patients hospitalisés pour AVC ischémique $(\mathbf{n}=151)$ et de sujets contrôles $(n=111)$ par trois méthodes différentes: les anticorps anticardiolipine par ELISA, le temps de céphaline et le VDRL. Résultats: L'âge moyen des cas d'AVC ischémique était de 68 ans (de 29 à 91 ans) et celui des contrôles de 63 ans (de 29 à 86 ans). La prévalence d'AAPL détectés par au moins une des trois méthodes était de $12 \%$ pour les cas d'AVC ischémique et de $10 \%$ pour les contrôles. Après correction pour les facteurs de risque connus tels l'âge, le sexe, le diabète, la maladie cardiaque, l'hypertension et la tabagisme, le risque relatif de l'AVC s'abaissait à 0.8 (IC. 0.4 à 1.2). Conclusion: Nos observations suggèrent que les AAPL ne seraient pas un facteur de risque indépendant de l'AVC ischémique chez des patients non sélectionnés qui ne sont pas connus comme porteurs d'un lupus érythémateux ou d'une sclérodermie systémique. Cependant, une évaluation plus poussée du rôle de l'anticoagulant lupique est justifiée.

Can. J. Neurol. Sci. 1998; 25: 64-69

In 1986, stroke accounted for $7.6 \%$ of deaths in Canada' and substantial personal and economic costs due to resulting disabilities. More than $85 \%$ of strokes are ischemic, with the rest being primary intracerebral hemorrhage or subarachnoid hemorrhage. ${ }^{2}$ With the identification and treatment of some of the major risk factors for stroke, there has been a decline in the incidence of stroke over the past few decades. A 1993 multicenter controlled study of 248 ischemic stroke (IS) patients that adjusted for age, gender, smoking, hypertension, cardiac disease, and diabetes mellitus ${ }^{3}$ sug- gested that anti-cardiolipin antibodies (ACA) are an independent risk factor for first ischemic stroke. If this is confirmed, identification of people at risk and subsequent intervention may lead to a further reduction in the incidence or morbidity of stroke.

From the Department of Clinical Neurosciences, Foothills Hospital, University of Calgary.

RECEIVED FEBRUARY 27, 1996. ACCEPTED IN FINAL FORM JULY 21, 1997.

Reprint requests to: L.M. Metz, 12th Floor, Neurology, Foothills Hospital, 1403 - 29th Street N.W., Calgary, Albena, Canada T2N 2T9 
Interest in the role of antiphospholipid antibodies (APLA) as a potential contributing factor to ischemic stroke (IS) has been heightened by several studies over the past 10 years. ${ }^{3-12}$ Prior to 1987, fewer than 70 patients with IS and APLA had been reported..$^{13}$ Since then, a number of studies have demonstrated that the prevalence of anticardiolipin antibodies, one of several APLA, ranges from $6.8 \%$ to $46 \%$ in IS and TIA patients. ${ }^{6-12}$ The prevalence of APLA reported in controls varies between 0 and $12 \%,{ }^{3.6-10,14}$ and like other autoantibodies, the frequency increases with age ${ }^{15}$ and is correlated with the presence of other autoantibodies. ${ }^{14}$ Some studies included both IS and TIA patients; $5,7,9,12$ many assessed only patients within certain age groups ${ }^{7,11}$ or their study population had an average age less than sixty years; ${ }^{5,6,9,10}$ and several studies included patients with autoimmune diseases including systemic lupus erythematosis where the prevalence of antiphospholipid antibodies is known to approach 30\%..$^{5-7,9-11}$ Most control populations studied represented cohorts of hospitalized patients or unmatched blood donors. Different measures of APLA (ACA, VDRL, and lupus anticoagulant) and different laboratory methods to detect ACA have been used. Thus, wide variation in the prevalence of APLA in both stroke and control populations is likely, at least in part, to be related to different patient and control selection criteria and different laboratory methods.

The studies by the Antiphospholipid Antibody in Stroke Study (APASS) group ${ }^{3}$ and Chakravarty et al. ${ }^{8}$ appear to be based on unselected ischemic stroke patients without known collagen vascular disease but the methods of evaluating the size and demographic features of missed patients to determine if their samples were truly unselected is not reported. It is possible that including only IS patients admitted to American tertiary referral centres during "regular working hours" 3 may lead to over-representation of referred patients, and therefore result in a "selected", potentially biased, population. This potential for bias is less likely to be an issue in the British Medical System where Chakravarty et al. ${ }^{8}$ completed their study. Most Canadian tertiary care hospitals also function as community hospitals and Canadians generally have little choice in where they obtain their medical care in emergent and urgent situations. This lends itself to relatively unbiased patient selection.

Our objective was to undertake a case-control study to determine the frequency of APLA (ACA, false-positive VDRL tests, or a prolonged APTT) in unselected, hospitalized IS patients compared to age and gender matched controls. In addition, we collected data on other stroke risk factors in order to determine the odds ratio and adjusted odds ratio for stroke occurring in individuals with APLA.

\section{MeThODS}

\section{Subjects}

Over a 16 month interval 151 IS patients who were assessed by the neurology service at two teaching hospitals were prospectively evaluated. Diagnosis was confirmed by the treating neurologist and was based on history, physical examination and cranial computerized tomography. Patients were excluded if consent could not be obtained, if serum could not be obtained within 5 days of the ischemic event, or if they had known systemic lupus erythematosus, Sjögren's syndrome, or systemic sclerosis. Patients with previous strokes were not excluded, but were identified for subsequent stratified analysis. Both hospitals function as both primary and tertiary care centres within their region.

Demographic information, risk factor data (hypertension, diabetes, cardiac disease, smoking, previous TIA or stroke, hyperlipidemia, vascular claudication, and drug and alcohol abuse), description of the stroke (symptoms, neurologic deficit, disability, and investigations), and conditions associated with SLE and the antiphospholipid syndrome were obtained by chart review and a structured interview undertaken by the study neurologist or the stroke study nurse. When necessary, this information was obtained from family members.

Patients were asked to refer a similar aged $( \pm 5 \mathrm{yr})$ friend, family member or, when necessary, spouse to participate in the study as controls. Same gender controls were preferred and those with a previous history of stroke were excluded. Controls gave informed consent, provided historical information identical to that of the study patients (except for the stroke data) by a structured questionnaire, and serum was obtained.

To determine if there was selection bias in our stroke group medical records of all patients discharged during the study period with a diagnosis of stroke were reviewed. The number of patients who were excluded or not evaluated, the reason they were excluded or not evaluated, and their age and gender were determined.

\section{Data Analysis}

Fisher's exact test and chi-square analysis were used for the comparison of categoric variables (gender, ethnic origin, education level, smoking, hypertension, cardiac disease, and diabetes mellitus). The $t$ test was used for analysis of age. Logistic regression, with hierarchical adjustment for known risk factors for stroke (age, gender, smoking history, diabetes mellitus, cardiac disease, and hypertension) was used to assess the relative contribution of APLA, over and above these risk factors. APLA were analysed as a composite score which represented the summary of any positive value from either the ACA, the lupus anticoagulant (APTT), or the VDRL test. All statistical tests were performed with SAS 6.08 (Windows Version for PC).

\section{Preparation and Storage of Serum Specimens}

Blood was collected without an anticoagulant and allowed time to clot at room temperature. The specimen was then centrifuged at $2000 \mathrm{rpm}$ for 5 minutes at room temperature to sediment cellular elements and the clot. The serum was removed and heated at $56^{\circ} \mathrm{C}$ for 30 minutes to inactivate complement and particulate debris was clarified by additional recentrifugation at $14000 \mathrm{rpm}$ for 10 minutes in an Eppendorf centrifuge (Model 5415C). Control sera to establish normal cutoff values for the ACA ELISA described below were obtained from the serum bank at The University of Calgary. ${ }^{14}$

\section{ELISA for Cardiolipin Antibodies}

The protocols for the detection of cardiolipin antibodies followed recommendation of the Second World Conference on Phospholipids. ${ }^{16}$ All ELISA reagents were prepared in Nanopur (Millipore Corp.) water and the buffers were prepared immediately prior to performing the procedure. Phosphate buffered saline (PBS) was prepared by combining $8.2 \mathrm{~g} \mathrm{NaCl}, 20 \mathrm{ml} 0.5$ 
$\mathrm{M}$ phosphate buffer, $\mathrm{pH} 7.4$, and $0.1 \mathrm{~g}$ thimerosal in a final volume of 1 litre distilled water. A known positive control was serially diluted from $1 / 200$ to $1 / 1600$ in $10 \%$ newborn calf serum (NBCS) (Gibco) in PBS. Two known positive and two known negative controls were diluted to $1 / 200$ in $10 \%$ NBCS/PBS. The sera of the stroke patients and their matched controls were diluted to $1 / 200$ in PBS. Cardiolipin (Sigma Chemical Co.) was prepared at a concentration of $5 \mu \mathrm{g} / \mathrm{ml}$ in ethanol. Fifty $\mu \mathrm{l}$ of the cardiolipin solution was pipetted into the wells of Immunion I microtiter plates (Gibco). To determine the background binding of each serum, "blank" wells were filled with $50 \mu \mathrm{l}$ of ethanol in the absence of cardiolipin. After the ethanol was evaporated overnight at $4^{\circ} \mathrm{C}$, the plates were blocked with $300 \mu \mathrm{l}$ of a $10 \%$ solution of NBCS in PBS, and placed at $4^{\circ} \mathrm{C}$. The plates were then washed 3 times with $300 \mu \mathrm{l}$ of a $5 \%$ NBCS/PBS solution. Two hundred $\mu$ l of the dilutions of standards, controls, and test specimens were added to the appropriate wells and the plates were incubated at $4^{\circ} \mathrm{C}$ ovemight.

Each plate was washed 3 times with 5\% NBCS/PBS solution. A polyvalent (IgG, IgA, IgM) anti-human antibody conjugated to alkaline phosphatase (Sigma Chemical Co.) was diluted to $1 / 1400$ in $10 \%$ NBCS/PBS, and $200 \mu l$ of the conjugate were added to each well and the plate incubated for 90 minutes at room temperature with constant agitation. The excess conjugate was removed by washing the plates 5 times with $5 \%$ NBCS/PBS. Two hundred $\mathrm{ml}$ of a developing solution (1 $\mathrm{mg} / \mathrm{mL}$ of p-nitrophenylphosphate in diethanolamine buffer (105.14 $\mathrm{g}$ diethanolamine, $0.102 \mathrm{~g} \mathrm{MgCl}_{2} \cdot 6 \mathrm{H}_{2} \mathrm{O}$, to a volume of 1 litre with distilled water and brought to a $\mathrm{pH} 9.8$ with concentrated $\mathrm{HCl}$ ) was added to each well. The optical density (OD) was read at $405 \mathrm{~nm}$ on the Titretek Multiscan photometer (Flow Laboratories). The time interval for the controls to reach their respective $O D$ values was 12 to 15 minutes. The optical density units for each result were plotted on a curve generated by the known positive sample results included on each plate. A negative result was defined as less than $10 \mathrm{GPL}$. Ten-eleven GPL was reported as borderline, 11-20 GPL as low positive, 20-100 GPL as medium positive, and $>100$ as high positive.

\section{Venereal Diseases Research Laboratory (VDRL) Test for Syphilis}

The VDRL test ${ }^{17}$ was performed using reagents (VDRL antigen, VDRL-Buffered Saline) and reactive (R), weakly reactive (w) and nonreactive ( $N$ ) serum samples from Fisher Diagnostics. The VDRL antigen suspension was prepared fresh each testing day as follows. Four hundred $\mu$ of a VDRL phosphatebuffered saline solution was pipetted into a $30 \mathrm{ml}$ glass stoppered vial. One-half $\mathrm{ml}$ of VDRL antigen was added directly to the saline solution while continuously rotating the vial on a flat surface. The antigen was added dropwise at a rate of $0.5 \mathrm{~mL}$ of antigen per 6 seconds. Then, $4.1 \mathrm{ml}$ of buffered saline was added, the vial was capped and rapidly inverted 30 times.

Fifty $\mu l$ of serum were placed into one ring of a paraffin or ceramic-ringed slide. The VDRL suspension was gently resuspended. One free falling drop $(-20 \mu \mathrm{l})$ of antigen was added to each circle containing serum. The slide was then placed on a mechanical rotator for 4 minutes at $180+2 \mathrm{rpm}$ in a covered moist humidifying chamber to prevent evaporation. Using a $10 \mathrm{X}$ objective and a 10X ocular, the slide was read under bright field microscopy and the results recorded according to the size of clumps. Medium or large clumps were reported as reactive (R), small clumps as weakly reactive ( $\left.\mathrm{CA}^{\prime}\right)$, and no clumping or very slight roughness as nonreactive $(\mathrm{N})$.

\section{Activated Partial Thromboplastin Time (APTT)}

Standard APTT was performed by incubating $0.1 \mathrm{ml}$ of plasma with $0.1 \mathrm{ml}$ of automated APTT (Organon Teknika, Durham, $\mathrm{NC}$ ) for $5 \mathrm{~min}$ at $37^{\circ} \mathrm{C}$, after which $0.1 \mathrm{ml}$ of $25 \mathrm{mM} \mathrm{CaCl}_{2}$ was added and the clotting time recorded. The presence of a lupus anticoagulant (LA) was not confirmed in all patients.

\section{Results}

During the 16 month study period 379 IS patients without known connective tissue disease were admitted to hospital. One hundred fifty-one IS patients and 111 controls were studied (Table 1). Cases and controls differed for age, gender, and race. On average, the age of IS cases (68 years) was approximately 5 years older than controls (63 years) $(p<.01)$, but the age range was similar (29 to 91 years for cases and 29 to 86 years for controls). Thirty-seven per cent of cases and $57 \%$ of controls were female $(\mathrm{p}<0.01)$. There were more non-Caucasians in the case group ( $10 \%-3$ black, 3 native Canadian, 10 other race) than in controls $(1.8 \%)$ ( $\mathrm{p}<.05$ by Fisher exact test). The age difference and the larger number of females in the control group was due to the method of recruitment for controls. Many elderly male IS patients did not have a same gender friend or family member to refer who was within 5 years of their age. This resulted in referral of a high number of spouses, who were often younger and female, or in referral of no control. Racial differences were also related to the method of recruitment for controls because many non-Caucasians did not wish to refer a friend or relative. This may be related to different cultural perspectives of health and/or medical research.

Two hundred twenty-eight ischemic stroke patients admitted to hospital during the study period were not enrolled in the study (Table 2). 112 patients were missed because they were not referred for this study within 5 days of the stroke. Their mean age was 70 years and $45 \%$ were female. They did not differ statistically from study patients. 116 patients were excluded: 51 who were never assessed by a neurologist, 28 who were assessed by a neurologist more than 5 days after stroke onset, 14 who died prior to enrollment, 19 from whom informed consent could not be obtained within 5 days, and 4 patients who refused to enter the study. The average age of the enumerated but ineligible

Table 1: Demographic and Clinical Features of IS Patients and Controls.

\begin{tabular}{|c|c|c|c|}
\hline & $\begin{array}{l}\text { Patients } \\
(n=151)\end{array}$ & $\begin{array}{l}\text { Controls } \\
(\mathrm{n}=111)\end{array}$ & $\begin{array}{l}\text { Odds Ratio } \\
(95 \% \mathrm{Cl})\end{array}$ \\
\hline Mean Age (SD) & $67.75(12.80)$ & $62.75(11.75)^{*}$ & \\
\hline Gender \% Female & 37 & 57 & $2.24(1.36-3.69)$ \\
\hline Race \% non-Caucasian & 10 & $1.8^{* *}$ & \\
\hline \multicolumn{4}{|l|}{ Risk Factors } \\
\hline \% smoking & 71 & 47 & $2.83(1.70-4.72)^{* *}$ \\
\hline$\%$ cardiac disease & 45 & 13 & $5.56(3.02-10.24)^{*}$ \\
\hline$\%$ diabetes mellitus & 19 & 3 & $8.40(2.94-23.97)^{*}$ \\
\hline$\%$ hypertension & 64 & 26 & $5.20(3.07-8.80)^{*}$ \\
\hline $\begin{aligned} * \mathrm{p} \text { value }< & =0.01 \\
* * \mathrm{p} \text { value }< & =0.05\end{aligned}$ & & & \\
\hline
\end{tabular}


Table 2: Age and Gender of IS Patients Enrolled, Excluded or Not Referred for Inclusion.

\begin{tabular}{|c|c|c|}
\hline & Age & Gender \\
\hline Patients $(n=151)$ & 67.75 years & $37 \%$ female \\
\hline $\begin{array}{l}\text { Excluded IS patients ( } n=116) \\
\text { never assessed by a neurologist }(51) \\
\text { assessed more than } 5 \text { days after stroke (28) } \\
\text { death prior to enrollment (14) } \\
\text { informed consent could not be obtained (19) }\end{array}$ & & \\
\hline refused (4) & 77 years & $62 \%$ female \\
\hline Unreferred IS patients (not enrolled) $(n=112)$ & 70 years & $45 \%$ female \\
\hline
\end{tabular}

patients was 77 years and $62 \%$ were female. Patients who were not seen by a consulting neurologist or died prior to consent were older ( 79 years and 76 years respectively). These patients were significantly older. The $p$-value is $<.001$ for the patients not seen by the neurologist (SD 12.8) and 0.008 for those who died (SD 12.7).

Significant differences between cases and controls were found for each of the established risk factors. Odds ratios ranged from 2.8 to 8.4 (Table 1). Cases had higher smoking rates $(71 \%$ vs. $47 \%)$, more cardiac disease $(45 \%$ vs. $13 \%)$, and greater prevalence of both diabetes mellitus (19\% vs. $3 \%$ ), and hypertension (64\% vs. $26 \%$ ). Forty patients had previous stroke or TIA (see Table 3). When the above risk factors were analysed, no significant differences between these two subgroups of IS patients were found.

APLA were found in $12 \%$ of cases and $10 \%$ of controls (unadjusted odds ratio $1.3 ; \mathrm{p}=\mathrm{NS}$ ) (Table 4). The adjusted odds ratio for APLA, after correcting for age, gender, smoking, cardiac disease, diabetes mellitus, and hypertension was $0.8(95 \%$ $\mathrm{Cl}, 0.4$ to 1.5 ). When specific APLA were considered, there were no significant differences between IS cases and controls for ACA or VDRL; however a higher prevalence of prolonged APTT (7\% vs. $1 \%$ ) was found in stroke subjects. APTT was done in $98 / 151(65 \%)$ of cases and prolonged APTT was confirmed to be due to LA in 4 of 7 cases but not further evaluated 3 of 7 cases. The prevalence of prolonged APTT was greater in patients with prior stroke (12.5\%) compared to patients with no prior stroke (1.8\%).

Patients were more likely to have medium or high titres of ACA to polyvalent antibody (Table 5). Because others have

Table 3: Stroke Status in 151 Ischemic Stroke Cases.*

\begin{tabular}{lll}
\hline & $\begin{array}{l}\text { No Prior Stroke } \\
\mathbf{n = 1 1 1}\end{array}$ & $\begin{array}{l}\text { Prior Stroke or TIA } \\
\mathbf{n = 4 0}\end{array}$ \\
\hline $\begin{array}{l}\text { Gender female } \\
\text { Race non-Caucasian }\end{array}$ & $44(41)$ & $11(28)$ \\
Risk Factors & $12(11)$ & $3(8)$ \\
$\quad$ smoking & $76(70)$ & $29(73)$ \\
$\quad$ cardiac disease & $45(41)$ & $23(58)$ \\
$\quad$ diabetes mellitus & $19(17)$ & $10(25)$ \\
$\quad$ hypertension & $73(67)$ & $22(55)$ \\
\hline
\end{tabular}

* numbers in parentheses are \%
Table 4: Antiphospholipid Antibodies (APLA) in Ischemic Stroke Patients and Controls.

\begin{tabular}{lllll}
\hline & APLA & ACA & VDRL & APTT** \\
\hline Ischemic Stroke & $18 / 151(12)$ & $12 / 151(8)$ & $2 / 150(1.3)$ & $7 / 98(7)$ \\
- Prior Stroke & $4 / 40(10)$ & $4 / 40(10)$ & $0 / 40(0)$ & $5 / 40(12.5)$ \\
- no Prior Stroke & $7 / 111(6.3)$ & $8 / 111(7.2)$ & $2 / 10(1.8)$ & $2 / 110(1.8)$ \\
Control & $11 / 111(10)$ & $9 / 109(8.3)$ & $4 / 109(3.7)$ & $1 / 102(1)$ \\
\hline
\end{tabular}

* numbers in parentheses are $\%$.

** LA confirmed in $4 / 7$ patients ( 2 with prior stroke) and $0 / 1$ controls

Abbreviations: $A P L A=$ antiphospholipid antibodies $(A C A+A P T T+$ VDRL); $A C A=$ anticardiolipin antibody; $V D R L=$ Venereal Diseases Reseanch Laboratory test; $A P T T=$ activated partial thromboplastin time.

generally included titres of $10 \mathrm{GPL}$ or more as low positive we have combined our borderline group ${ }^{10.11} \mathrm{GPL}$ with our low positive group to allow comparison. While more cases than controls have medium and high titres the numbers in our study, as in all previous studies, are too small to allow statistical analysis.

\section{Discussion}

This study provides evidence that APLA, and particularly ACA, may not be predictive of stroke in an unselected population. Although the prevalence rate for APLA in cases (12\%) was within the range of published data ${ }^{3.6-10.14}$ the prevalence rate in controls $(10 \%)$ was higher than the rate $(0-4.3 \%)$ in two studies that most closely resemble this one ${ }^{3,8}$ Our findings are consistent with a recent Canadian study ${ }^{18}$ where the prevalence of ACA and LA were determined in an unselected population undergoing investigation for venous thromboembolism (VTE). These authors reported that there was no association between the presence of ACA and occurrence of VTE due to the high frequency of positive ACA assays in patients without VTE. Our data are also in general agreement with the recent report of Ginsberg et al." who concluded that elevated ACA levels were not a risk factor for IS in healthy adult men.

Chakravarty et al. ${ }^{8}$ studied 100 IS and TIA patients over the age of 60 and found APLA in $21 \%$ of cases but in none of the controls. Their stroke population was older (75 years vs. 68 years) but if age was the factor determining APLA positivity there should have been a corresponding increase in APLA in

Table 5: Antiphospholipid Antibodies (APLA) Titre in Ischemic Stroke Patients and Controls.

\begin{tabular}{|c|c|c|c|c|c|}
\hline & $\begin{array}{l}\text { Our cases } \\
(\mathrm{N}=12)\end{array}$ & $\begin{array}{l}\text { Our } \\
\text { controls } \\
(n=9)\end{array}$ & $\begin{array}{l}\text { Brey cases } \\
(7) \\
(n=13)\end{array}$ & $\begin{array}{l}\text { Brey } \\
\text { controls (7) } \\
(n=1)\end{array}$ & $\begin{array}{l}\text { Hess cases } \\
(9) \\
(n=9)\end{array}$ \\
\hline low pos & 6 & 7 & & $1(\lg M)$ & 6 \\
\hline $\begin{array}{l}\text { med } \\
\text { pos }\end{array}$ & 3 & 1 & \multicolumn{2}{|c|}{$\begin{array}{l}8(\operatorname{IgG}) \\
3(\operatorname{IgG} \& \operatorname{IgM})\end{array}$} & 2 \\
\hline $\begin{array}{l}\text { high } \\
\text { pos }\end{array}$ & 3 & 1 & $2(\lg M)$ & l(IgM) & 1 \\
\hline
\end{tabular}


their controls. Their very low rate in controls was not in keeping with other published results.

A previous report by the APASS group ${ }^{3}$ of similar case-control design, but using multicenter accrual, found a 2.34 fold relative risk of stroke in ACA positive patients $(9.7 \%$ in cases vs. $4.3 \%$ in controls), which did not change significantly after adjusting for established risk factors. In our study multiple logistic regression, which incorporated an adjustment for other risk factors such as smoking, diabetes, and previous cardiac disease the adjusted risk attributable to APLA alone was found to be less than one $(0.8 ; \mathrm{Cl}=0.4$ to 1.5$)$. The APASS controls were drawn from hospitalized patients without stroke, the majority of whom had had surgery. The most significant differences between cases and controls in their study were in the ethnic origin of patients ( $p=0.001$; more blacks and Hispanics) and in the occurrence of hypertension $(p=0.001)$ and diabetes mellitus $(p=0.007)$ both of which have been linked to ethnic origin in other studies. Smoking and coronary artery disease were similar in cases and controls. The mean age of patients in their study was 66 years, similar to the mean age of 68 in our study. Our study had far fewer blacks and no Hispanics, but had higher rates of smokers and those with prior cardiac disease, hypertension, and diabetes mellitus in the IS group.

The major difference in our results is that our controls had a higher rate of APLA positivity than in the APASS study. Selection of controls in our study was deliberately directed to individuals who would likely share the same social environment as cases (spouses, friends, or attending family members). It is conceivable that a common environmental stimulus triggered the antibody formation in both cases and controls, thus elevating the antibody detection rate in controls. Studies which select hospitalized patients may not account for this potential factor. On the other hand, given that three methods (ACA, VDRL, and prolonged APTT) were used to detect antiphospholipid antibodies it may be that this higher rate could be expected for a population of this age distribution. A second possibility is that there is an association between APLA status and race. It may be that the increased incidence of APLA positivity in the APASS study group was related to racial differences between their study population and controls. Third, a statistical correlation between APLA status and other risk factors such as cardiac disease, diabetes mellitus, hypertension, or smoking may have reduced the APLA contribution after this correlation was accounted for in the multiple logistic regression hierarchical model. Our study had significant differences at the univariate level in each of these factors whereas the APASS study had differences only in hypertension and diabetes mellitus, thus providing a potential differential effect during the regression modelling. Finally, selection bias may have been present in their study population that would reduce the generalizability of their results. Their sample may have been drawn from a more selected population which may have been biased toward increased frequency of APLA in cases. They recruited 255 cases from 15 large urban American referral centres over 5 months ( 3.4 cases/ centre/ month). We recruited 151 cases from 2 large urban community/ referral centres over 16 months (4.7 cases/ centre/ month). Our faster rate of accrual, despite smaller urban size, supports our belief that our sample is less selected and is therefore less likely to be affected by recruitment bias. The lack of difference between their cases and controls for known risk factors for stroke (cardiac disease and smoking) also suggests a slightly atypical stroke population.

Our study also had significant age and gender differences between cases and controls. Controls were younger and more likely to be female. It is unlikely that the high rate of APLA in controls is due to their younger age because all prior data suggest the opposite. While it is possible that the higher proportion of women in the control group (57\%) is responsible for the increased frequency of APLA in controls, gender has not been associated with risk of APLA positivity if patients with collagen vascular disease are excluded. Other control populations, which have had a similarly high proportion of females (53-62\%), found APLA in $0-8 \%{ }^{7-9}$

Case-control studies have limitations with respect to possible sampling bias in recruiting patients and controls as well as in potential recall bias for relevant risk factors. This single center study attempted to recruit all consecutive patients admitted to 2 teaching hospitals with IS and while this was not achieved, analysis of the demographics of ineligible and missed patients suggests a random sample was obtained except for the very elderly, especially those who do not survive the first few days of their stroke. Ascertainment of diabetes, hypertension, and cardiac disease may also have been subject to recall bias. Although a standard instrument was used for both cases and controls family members had to provide historical information for a few patients. Over-reporting of risk factors by family members of a small number of patients was unlikely to cause the large difference in these variables between patients and controls. We therefore believe that the results of this study are generalizable to patients hospitalized in similar circumstances.

Laboratory factors may also be responsible for the discrepancy in our results. Recent studies ${ }^{19-21}$ have shown that purified APLA reacts with cardiolipin in the presence of a cofactor. The primary candidate for this cofactor is $\beta_{2}$-glycoprotein 1 (apolipoprotein $\mathrm{H}$ ) (reviewed in ${ }^{21}$ ). Since our assay utilized newborn calf serum, it is possible that our observations were also dependent on $\beta_{2}$-glycoprotein 1 . However, considering the lack of a statistically significant difference between the IS population and normal controls, we do not expect the presence or absence of a cofactor in our assay to be a confounding factor. We used polyvalent (IgG, IgA, IgM) anti-human antibody. Polyvalent antibody detects the presence of all types of cardiolipin antibody. Although the APASS group found a statistically significant difference between cases and controls only for the presence of the IgG isotype $(p=0.018)$ small numbers made it impossible for them to address the importance of isotype distribution. There is no consistent evidence that any isotype is more significant than any other. Low titres of APLA may also prove to be insignificant as they have been reported to often be transient. There are currently insufficient data to determine the significance of this factor.

Possibly combining positive results from all three tests for APLA (ACA, VDRL, APTT) has led to a false negative conclusion. The marked difference in the frequency of abnormal APTT between our cases $(7 \%)$ and controls $(1 \%)$ raises this issue but the clinical significance of this finding is not clear for three reasons. First, only 98/15l (65\%) of cases had APTT done and a bias to patients with prolonged APTT may have been hidden. Second, prolonged APTT was confirmed to be due to LA in only 4 of 7 cases therefore potentially upwardly biassing the 
prevalence of LA in this population. Third, the prevalence of prolonged APTT was greater in patients with prior stroke $(12.5 \%)$ compared to patients with no prior stroke $(1.8 \%)$. Although we attempted to identify patients on anticoagulants, it is possible that this information was not reported by patients or recorded in the chart and the increased prevalence of prolonged APTT may be accounted for by therapeutic modalities prior to admission. Alternatively, these data are consistent with a recent study suggesting that only prolonged APTT is associated with increased risk of venous thromboembolism. ${ }^{18}$ The increased prevalence in those with prior stroke may then be clinically significant.

The possibility of a Type II error needs to be considered in studies such as ours which suggest negative results. Assuming a baseline APLA positive rate of $10 \%$, our study would detect a 2.5 fold increased risk with $90 \%$ power. ${ }^{22}$ A decision to stop enrollment was made after preliminary assessment of the APLA positive rate $(12 \%)$ on 151 patients was determined. The control rate at this time was $9.9 \%$ and it was assumed that there was insufficient justification to enroll more patients for the sole purpose of increasing power to detect the small, clinically irrelevant unadjusted odds that was evident at the time.

In summary, in contrast to some previous studies, our study fails to uphold the view that APLs, particularly ACA, are a significant risk factor for stroke in an unselected population but suggests LA may be important. Known risk factors for stroke including hypertension, previous cardiac disease, diabetes, and smoking history were confirmed to be significant predictors of stroke in our study population. Multivariate analysis which includes these factors decreases the significance of APLA in the prognostic equation. Future studies should carefully consider the importance of LA and ensure that the control population targeted is better matched for race, environment, and gender than we or others have yet achieved. Patient-referred controls is not a reliable method of obtaining matched controls in this population. Detailed analysis of the time sequence of antibody formation in the period immediately following stroke would also be helpful. Adjustment for established risk factors should be performed as a routine.

\section{ACKNOWLEDGEMENTS}

The authors thank Ms. Hollis Richter, Mrs. Annie Fong, and Mrs. Maureen Robertson for technical assistance. The database management of Rosario Talavera is appreciated. This research was supported by a grant from MSI (Medical Services Incorporated), the Medical Research Council of Canada (Grant \#MA6400) and the Toupin Foundation.

\section{REFERENCES}

1. Statistics Canada. Causes of Death Vital Statistics Volume IV, Catalogue. 1986; 84-203.
2. Sahs AL, Hartman EC, Aronson SM. Stroke: Cause, Prevention, Treatment and Rehabilitation. London, Castle House Publication Ltd., 1973; 1-24.

3. The Antiphospholipid Antibodies in Stroke Study (APASS) Group. Anticardiolipin antibodies are an independent risk factor for first ischemic stroke. Neurology 1993; 43: 2069-2073.

4. Briley DP, Coull BM, Goodnight SH. Neurological disease associated with antiphospholipid antibodies. Ann Neurol 1989; 25: 221-227.

5. Trimble M, Bell DA, Brien W, et al. The antiphospholipid syndrome: prevalence among patients with stroke and transient ischemic attacks. Am J Med 1990; 88: 593-597.

6. Kushner MJ. Prospective study of anticardiolipin antibodies in stroke. Stroke 1990; 21: 295-298.

7. Brey RL, Hart RG, Sherman DG, Tegeler CH. Antiphospholipid antibodies and cerebral ischemia in young people. Neurology 1990; 40: 1190-1196.

8. Chakravarty KK, Byron MA, Webley M, et al. Antibodies to cardiolipin in stroke: association with mortality and functional recovery in patients without systemic lupus erythematosus. Q J Med 1991; 397-405.

9. Hess DC, Krauss J, Adams RJ, et al. Anticardiolipin antibodies: a study of frequency in TIA and stroke. Neurology 1991; 41: 525528.

10. Montalban J, Codina A, Ordi J, et al. Antiphospholipid antibodies in cerebral ischemia. Stroke 1991; 22: 750-753.

11. Nencini P, Baruffi MC, Abbate R, et al. Lupus anticoagulant and anticardiolipin antibodies in young adults with cerebral ischemia. Stroke 1991; 23: 189-193.

12. Ginsberg KS, Liang MH, Newcomer L, et al. Anticardiolipin antibodies and the risk for ischemic stroke and venous thrombosis. Ann Intern Med 1992; 117: 997-1002.

13. Levine SR, Welch MA. The spectrum of neurologic disease associated with antiphospholipid antibodies. Arch Neurol 1987; 44: 877-883

14. Fields RA, Tourbeh H, Searles RP, Bankhurst AD. The prevalence of anticardiolipin antibodies in a healthy elderly population and its association with antinuclear antibodies. J Rheumatol 1989; 16: 623-625.

15. Fritzler MJ, Pauls JD, Kinsella TD, Bowen TJ. Antinuclear, anticytoplasmic and anti-Sjögren's syndrome antigen-A (SS-A/Ro) antibodies in female blood donors. Clin Immunol Immunopathol 1985; 36: 120-128.

16. Harris EN, Gharavi AE, Patel SP, Hughes GRV. Evaluation of the anticardiolipin antibody test: report of a standardization workshop held April 4, 1986. Clin Exp Rheumatol 1987; 3: 349-387.

17. Larsen SA, Hunter EF, Kraus SJ. A Manual of Tests for Syphilis Washington: American Public Health Association, 1990; 77-91.

18. Ginsberg JS, Wells PS, Brill-Edwards P, et al. Antiphospholipid antibodies and venous thromboembolism. Blood 1995; 86(10): 3685-3691.

19. McNeil HP, Simpson RJ, Chesterman CN, Krilis SA. Anti-phospholipid antibodies are directed against a complex antigen that includes a lipid-binding inhibitor of coagulation; $\beta$,-glycoprotein 1 (apolipoprotein H). Proc Natl Acad Sci 1990; 87: 120-124.

20. Galli M, Comfurius $P$, Maassen $C$, et al. Anticardiolipin antibodies (ACA) directed not to cardiolipin but to a plasma protein cofactor. Lancet 1990; 335: 1544-1547.

21. Koike T. Anticardiolipin antibodies and $\beta_{2}$-glycoprotein I. Clin Immunol Immunopathol 1994; 72: 187-192.

22. Altman, D.J. Practical Statistics in Medical Research. London: Chapman and Hall, 1991: 169-171. 